\title{
Open Set Anomaly Classification
}

\author{
Marcel Dix \\ Industrial Data Analytics \\ ABB Corporate Research Center \\ Ladenburg, Germany
}

\author{
Reuben Borrison \\ Industrial Data Analytics \\ ABB Corporate Research Center \\ Ladenburg, Germany
}

\begin{abstract}
Anomaly classification can provide a discrimination of the type of anomaly at hand, that can help the user to narrow down the search space during troubleshooting. A limitation of most machine learning (ML) classification models is being under the closed set assumption and thereby failing to recognize unknown data. Literature refers to this issue as the Opens Set Recognition (OSR) problem. In this paper, the given use case is the detection of technical faults in HVAC systems in commercial buildings. Here, the OSR problem is of high relevance, because it is very unrealistic that all possible faults are already known beforehand. This paper proposes an ML pipeline for open set anomaly classification. To address the OSR problem, the pipeline makes use of three common neural network architecture models that successively build on each other, namely two autoencoders and one ANN classifier. The advantage of using common well-known models is the ease of implementation for solution developers. The validation is made against an HVAC dataset published by LBNL that contains 7 fault cases, where we assume 3 of them as unknown. Our validation results show that the proposed pipeline can significantly reduce the risk of false class predictions in the presence of unknown data.
\end{abstract}

\section{CCS CONCEPTS}

- Computing methodologies $\rightarrow$ Neural networks; Supervised learning by classification.

\section{KEYWORDS}

Anomaly Detection, Open set recognition, HVAC, Smart Buildings

\section{ACM Reference Format:}

Marcel Dix and Reuben Borrison. 2021. Open Set Anomaly Classification. In The 8th ACM International Conference on Systems for Energy-Efficient Buildings, Cities, and Transportation (BuildSys '21), November 17-18, 2021, Coimbra, Portugal. ACM, New York, NY, USA, 4 pages. https://doi.org/10. $1145 / 3486611.3491118$

\section{INTRODUCTION}

To enable high yield in industrial systems and assets, it is important to maintain a desirable performance in these assets [1]. Faults can degrade an asset's performance and it is necessary to detect and diagnose faults (FDD) early on [1]. One way to achieve FDD is with

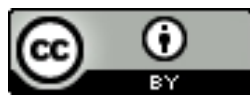

This work is licensed under a Creative Commons Attribution International 4.0 License. BuildSys '21, November 17-18, 2021, Coimbra, Portugal

(C) 2021 Copyright held by the owner/author(s).

ACM ISBN 978-1-4503-9114-6/21/11.

https://doi.org/10.1145/3486611.3491118 the help of machine learning (ML) algorithms, that are trained to detect and classify anomalous system behavior.

In this paper, open set anomaly classification problem is discussed at the example of HVAC (heating, ventilation, and air conditioning) systems for commercial buildings. It is a complex system consisting of several parts. The Air Handling Unit (AHU) is typically the most used component, but also error prone [2]. Here, data-driven fault detection and classification can help to reduce cost for maintenance and repair.

As for many technical systems and devices, there are known types of faults that can typically occur in an AHU [2][3]. For example, the valve of the heating or cooling coil may be leaking, or the damper may be stuck [4]. However, it is highly unlikely that all possible faults are known beforehand. There can always be a fault that has not been seen before.

A limitation of most ML classification algorithms is that they are under the closed set assumption, meaning, examples for possible faults have to be available beforehand for model training. This issue is typically referred to as the Open Set Recognition (OSR) problem in literature [5].

The contribution of this paper is to propose a feasible ML pipeline for open set classification in the context of anomaly detection. To address the OSR problem, the pipeline makes use of common neural network architecture models that successively build on each other. The advantage of using common models (for which there are, e.g., many code examples available) is the ease of implementation of the proposed OSR solution.

The remainder of this paper is structured as follows: Section 2 gives an overview of the related work in OSR. Section $3 \& 4$ present the proposed solution pipeline and discusses the evaluation results. Section 5 draws the conclusions and highlights our further research.

\section{RELATED WORK}

Machine learning has shown impressive results in classifying various types of data, such as image data or categorical data. As highlighted by Geng et al. in their survey paper on open set recognition [5], a limitation of most classification methods is that they are under the closed set assumption. However, in the presence of unknown data, the robustness of these classifiers weakens significantly.

The objective of OSR is to provide techniques for classifying data, that can reject/filter-out the unknown data samples while keeping the known samples to be classified accurately [6]. Recent studies on OSR have proposed various techniques for both statistical and deep learning classifiers.

Most statistical learning approaches follow a threshold-based scheme, as highlighted by Geng et al.[5]. For example, the authors in [7] [8] [9] follow the approach to decrease the region of known classes by modeling the space beyond a reasonable class support, 
which results in a threshold from where an unknown sample would enter the unknown open space.

OSR solutions for deep learning are the focus for this paper, because it has been observed in our recent studies [10] that neural networks do work well for classifying asset faults, but only if under the closed set assumption [11], which raises the question how to effectively reject unknown data.

Several techniques have been proposed how to embed OSR into a neural network architecture. E.g., Bendale and Boult [12] propose a novel activation function termed OpenMax to overcome the OSRlimitation of the commonly-used SoftMax. Furthermore, are several works, e.g., [13] [14] [6] [15], that propose a novel type of neural network architecture for classifiers that shall recognize and reject unknown data.

In this paper, the OSR problem is addressed with the help of an ML pipeline that makes use of three common neural network architecture models that build on each other in three successive steps. These models are two autoencoders and one ANN classifier. Using common well-known models as building blocks for the proposed OSR solution, rather than, e.g., proposing an own OSR model or model layer, has the advantage of an easy implementation.

\section{PROPOSED SOLUTION}

Figure 1 presents the proposed ML pipeline, using the formalized process description given in [16]. The pipeline is structured into two basic units: the detection of the fault itself (U1) and the classification of the type of fault under the open set assumption (U2).

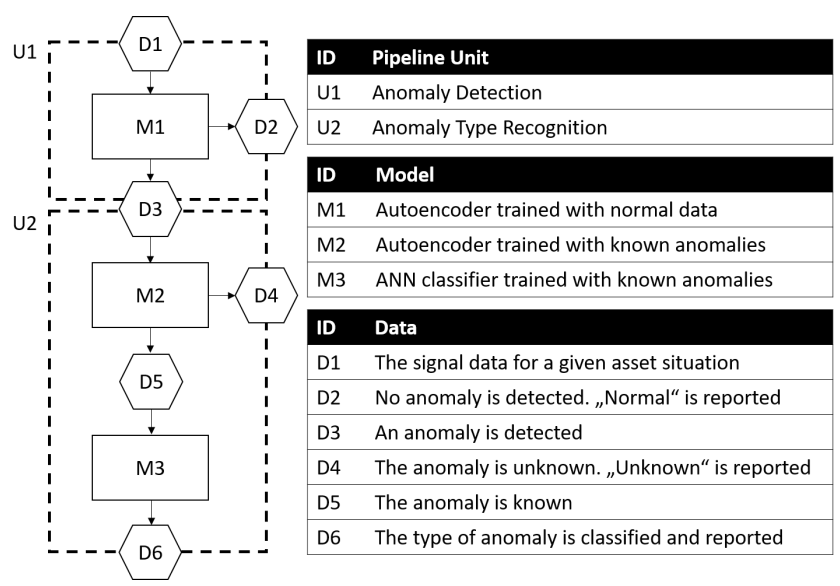

Figure 1: Proposed solution pipeline

In $\mathrm{U} 1$, the detection of an fault is realized with the help of an autoencoder model (M1). An autoencoder, as discussed by Yuan et al. in their survey paper [17], is a type of neural network that is useful for outlier detection. We chose this approach based on our recent studies [18] of comparing different approaches for detecting asset faults in the time series data from the assets. The model is trained to learn how to efficiently compress the data (D1) and reconstruct it back to its original form. In case of anomalous data that the model has not seen before, the reconstruction error is relatively high. This error provides a basis for an anomaly score. If this score is above a critical threshold, the given situation is reported as anomalous and potential fault (D3). The threshold is calculated during model training using the mean reconstruction error of all training samples, plus a sigma-factor of their standard deviation.

When a fault is detected, the goal of U2 is to classify the type of fault. The classification itself is realized with the help of an artificial neural network (ANN) classifier (M3) [19]. It is a model under the closed set assumption, which needs to be trained with known types of faults that can occur. For a given new fault that was detected in M1, the classifier tries to predict to which of its known classes this fault fits best.

To address the OSR problem, an additional autoencoder model (M2) is used. The aim of this model is to make a prediction if a given fault (D3) is known or not. This is achieved by training M2 with the same known failure data that is also used to train the classifier. As autoencoders are useful for outlier detection, an unknown fault will be recognized as an outlier by M2. When a given new fault is recognized as unknown, it will be rejected (D4). The fault-situations can still be reported to the user as an anomaly, but without making wrong assumptions about its type of fault. On the other hand, when the fault is recognized as known (D5) it can be passed on to the classifier (M3) to make a prediction about the type of fault (D6).

In summary, the need for three models in the proposed solution pipeline is explained as follows: Model M3 can classify anomalies, but fails to tell if a given anomaly is known or not. For this reason, model M2 is needed, which can recognize if an anomaly is known, but fails to tell if the given situation is anomalous in the first place. For this reason, model M1 is needed.

\section{EVALUATION}

To evaluate our approach, we use a public HVAC dataset that has been created by Lawrence Berkeley National Laboratory (LBNL) [4] by experimenting on a physical AHU system. The schematic of the AHU is shown in figure 2. The job of an AHU is to take in outside (fresh) air, filter it, cool/heat it and supply it through the ducts to the rooms. To ventilate the rooms, the air from the rooms is taken and a heat exchange takes place with the outside air, before the air is let out into the environment.

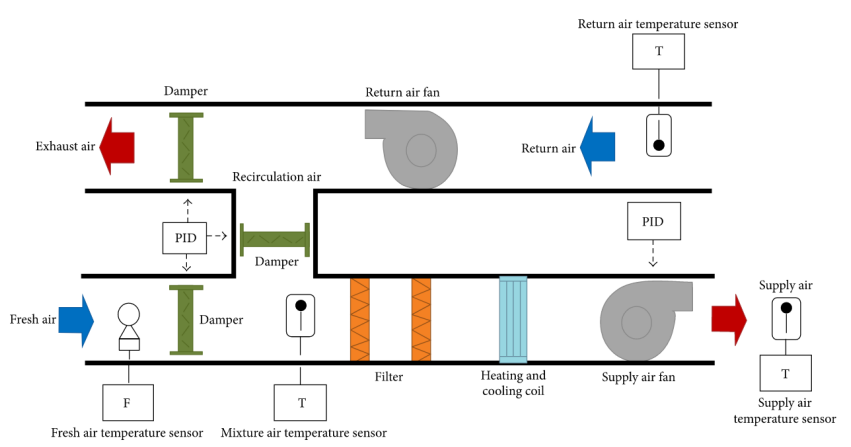

Figure 2: Schematic of AHU [20]

The dataset by LBNL consists of mainly temperature readings from different sensors of the AHU, namely sensors for the outside (fresh) air temperature (OAT), supply air temperature (SAT), return air temperature (RAT) and mixed air temperature (MAT). The dataset comprises a total of 11 days of operation of the AHU. 4 days are unfaulted. On the other 7 days, 7 different types of faults have 
been performed by the LBNL team on the AHU, such as leakages or a stuck damper. Each fault lasted for that whole day and then it was removed again at the end of the day. An overview of the faults, with their dates, and the methodology that was used to induce the fault, is given in figure 3 .

\begin{tabular}{|c|c|c|c|c|c|}
\hline \multicolumn{3}{|c|}{ Input Scenarios } & \multirow{2}{*}{$\begin{array}{l}\text { Known/ } \\
\text { unknown }\end{array}$} & \multirow[t]{2}{*}{ Method of fault imposition } & \multirow{2}{*}{$\begin{array}{l}\text { Fault } \\
\text { occurred } \\
\text { time }\end{array}$} \\
\hline Fault type & & Fault intensity & & & \\
\hline \multirow[t]{2}{*}{ OA Damper } & \multirow[t]{2}{*}{ Stuck } & Minimum position & Known & \multirow{2}{*}{$\begin{array}{l}\text { Automated override of control } \\
\text { signal values to indicate that } \mathrm{OA} \\
\text { damper is stuck. }\end{array}$} & 9/18/17 \\
\hline & & Fully open (100\%) & Known & & 9/19/17 \\
\hline \multirow{3}{*}{$\begin{array}{l}\text { Valve of } \\
\text { Heating Coil }\end{array}$} & \multirow[t]{2}{*}{ Stuck } & Fully open $(100 \%)$ & Known & \multirow{2}{*}{$\begin{array}{l}\text { Automated override of control } \\
\text { signal values to indicate that } \\
\text { heating coil valve is stuck. }\end{array}$} & $9 / 14 / 17$ \\
\hline & & Partially open (50\%) & Known & & 9/15/17 \\
\hline & Leaking & $\begin{array}{l}40 \% \text { of max coil } \\
\text { valve flow }\end{array}$ & Unknown & $\begin{array}{l}\text { Open heating coil bypass valve } \\
\text { to } 40 \% \text { of the maximum heating } \\
\text { coil valve flow. }\end{array}$ & $9 / 12 / 17$ \\
\hline \multirow[t]{2}{*}{$\begin{array}{l}\text { Valve of } \\
\text { Cooling Coil }\end{array}$} & Stuck & Fully open (100\%) & Unknown & $\begin{array}{l}\text { Automated override of control } \\
\text { signal values to indicate that } \\
\text { cooling coil valve is stuck }\end{array}$ & 9/22/17 \\
\hline & Leaking & $\begin{array}{l}50 \% \text { of max coil } \\
\text { valve flow }\end{array}$ & Unknown & $\begin{array}{l}\text { Open heating coil bypass valve } \\
\text { to } 50 \% \text { of the maximum heating } \\
\text { coil valve flow. }\end{array}$ & 9/11/17 \\
\hline \multicolumn{3}{|l|}{ Unfaulted } & N/A & N/A & $\begin{array}{l}\text { 9/20/17, } \\
9 / 21 / 17 \\
9 / 23 / 17 \\
9 / 24 / 17\end{array}$ \\
\hline
\end{tabular}

Figure 3: Evaluated AHU faults (adapted from [4])

To evaluate the OSR performance of the proposed pipeline, we made only 4 of the 7 fault cases known to the models M2 and M3 during model training. Figure 3 indicates which are these cases. The other 3 cases were left to be unknown to these models. To detect the faults as such, model M1 is used, which was trained with the 4 unfaulted days that represent normal AHU operation.

Figure 4 presents the performance of M1 to detect the 7 faults in the 11 operation days. Each plot represents one day, and shows the anomaly score across that day. When the score exceeds the given anomaly threshold (blue horizontal line), a fault is reported.
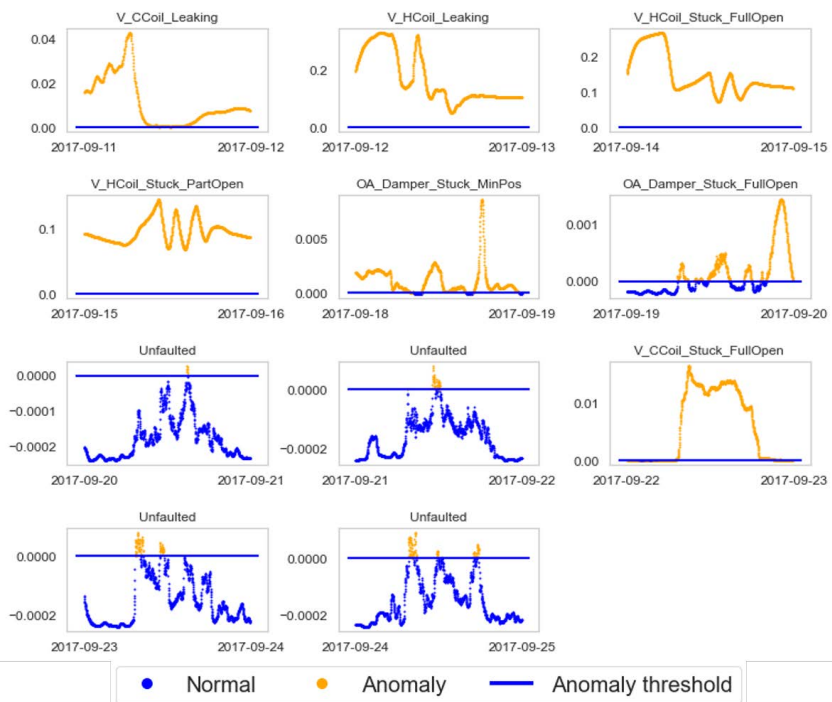

Figure 4: Evaluation of model M1 (7 faults and 4 non-faults)
It is observed in figure 4 that the overall performance of M1 to detect the 7 faults in the 11 days is high (accuracy $=91 \%$, precision $=97 \%$, recall $=89 \%$, and F1-score $=93 \%$ ). Some false positives can be observed on the unfaulted days, when the anomaly score exceeds the threshold very shortly. On 2017-09-19 (which is a damper fault) the score is less clearly above the threshold. This, however, can be explained by the absence of airflow sensors that typically provide much better signs for this type of faults.

For a given fault (D3) that was detected in M1, figure 5 shows the performance of our next model M2 to determine if this fault is known or not. The same visualization is used as in figure 4, as M2 is again an autoencoder.

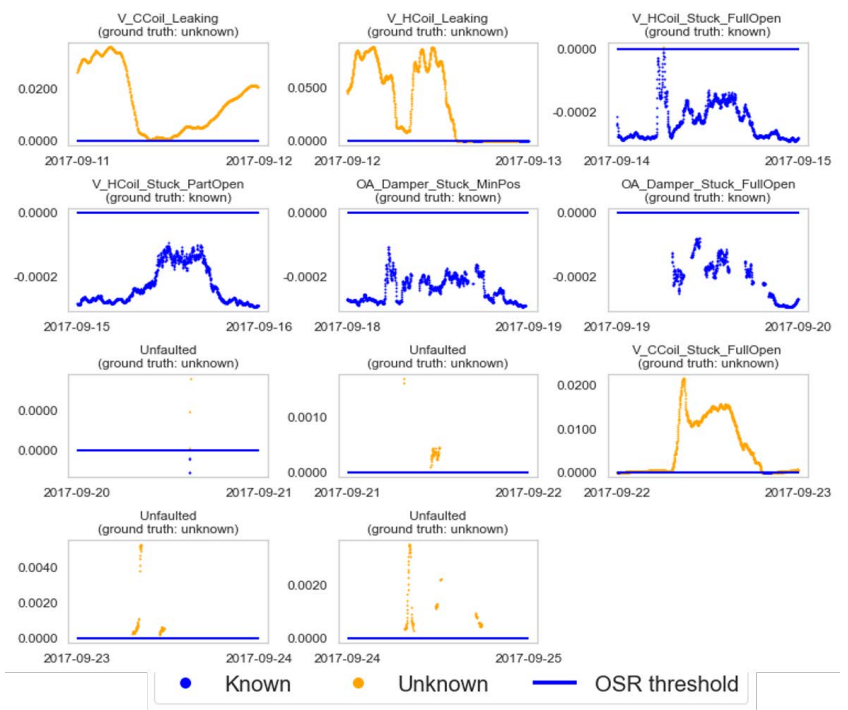

Figure 5: Evaluation of model M2 (4 known and 3 unknown cases. 4 unfaulted cases)

It is observed in figure 5 that all 7 fault cases are correctly recognized as known or unknown (accuracy $=95 \%$, precision $=94 \%$, recall=97\%, F1-score=95\%). Where M1 did not report a fault (D2), M2 does not make a prediction for known/unknown, which is shown by the gaps in the line plots of figure 5 . In the mentioned cases of figure 4 where M1 falsely predicted a few moments of the unfaulted days as anomalous, M2 does not recognize these as "known faults", which is good, because they are not faults at all.

Figure 6 presents the performance of the fault classification (M3) as confusion matrices. The axis labels indicate which are the 4 known and 3 unknown faults. The classifier makes a class prediction continuously at every minute of each fault, which results in the high number of predictions shown in figure 6.

For comparison, the left confusion matrix shows the performance without the recognition of unknown classes. The right matrix shows the performance that is achieved with the help of M2. In the left matrix, it is observed that for known cases M3 predicts the types of faults quite well (accuracy $=92 \%$ ). For unknown cases M3 does not make any predictions at all, because they are not known to the model. Instead, the model tries to fit them to any of its known classes. This behavior leads to a poor overall performance of M3, if no steps for OSR are taken. 


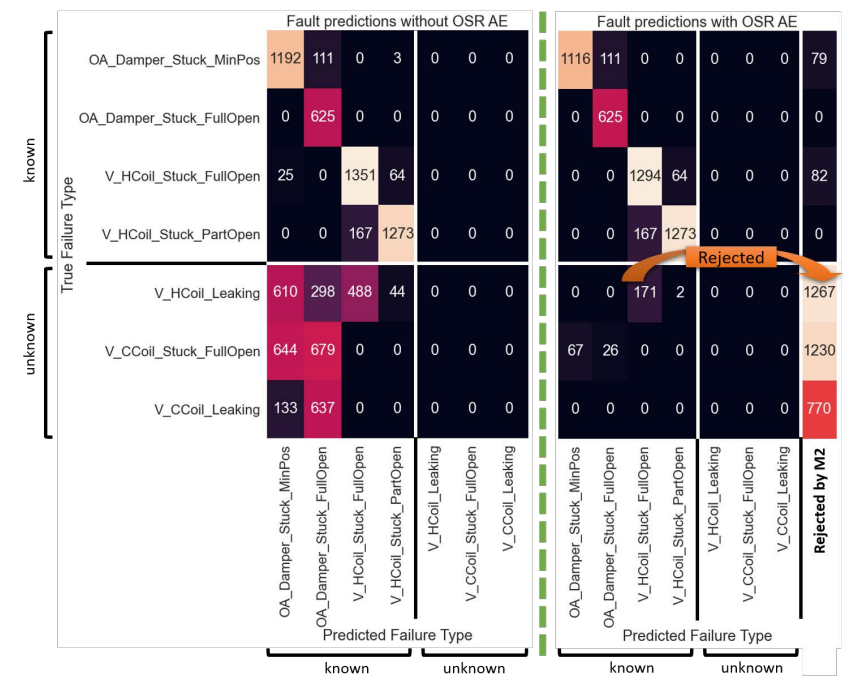

Figure 6: Evaluation of model M3: Without (left) and with (right) the rejection of unknown faults by M2

In the right matrix, with the help of M2, most of the unknown cases are correctly recognized as such and rejected. It is observed that not all unknown cases are recognized. Furthermore, some cases which are known are falsely rejected as unknown. But overall, the proposed solution was able to significantly reduce the risk of false classifications in the presence of unknown data. In our case of the evaluated HVAC dataset by LBNL, where we assumed 3 of the given 7 faults as unknown, this risk-reduction could be achieved by a magnitude of $88 \%$.

\section{CONCLUSIONS AND FUTURE WORK}

For many technical assets and systems there are known types of faults that can typically occur. A common troubleshooting practice by users is then to investigate if the root cause may be due to one of those known faults. Being able to correctly predict the type of fault at hand can help the user in this troubleshooting process.

A relevant limitation of most machine learning classifiers, that could be useful here for fault detection, is being under the closed set assumption. On the other hand, it is highly unlikely that all possible faults are known already beforehand when training the models. An unusual and not yet seen fault can always occur.

This paper presented a feasible ML pipeline to overcome the open set recognition problem in the context of anomaly detection. The pipeline makes use of common neural network models, and the OSR problem is addressed in the way how these models build on each other successively.

The validation was made against a publicly-available HVAC dataset that was created by the Lawrence Berkeley National Laboratory by experimenting on a physical AHU system. The dataset contains 7 fault cases in the AHU. For the purpose of this study, 3 of the 7 faults were assumed as unknown and only 4 cases were exposed to pipeline for model training. In the evaluation of this dataset it was observed that the proposed solution pipeline was able to reduce the risk of false class predictions related to unknown faults by $88 \%$.
A limitation of this study is the need to evaluate the solution against additional datasets, including assets from other industrial domains, to test how well the solution generalizes. First explorations of asset failures in process industries show promising results. However, an in-depth analysis is still ongoing. Our ongoing research is also to further extend the pipeline, in order to feed the rejected unknown faults back into the loop for model retraining including data labeling (active learning).

\section{ACKNOWLEDGEMENTS}

Part of this work was funded by the German Federal Ministry of Economics and Energy (BMWi) as per resolution of the German Parliament under the funding code 03EN1002D (DataFEE). The authors are responsible for the contents of this contribution.

\section{REFERENCES}

[1] You-Jin Park, Shu-Kai S Fan, and Chia-Yu Hsu. 2020. A review on fault detection and process diagnostics in industrial processes. Processes, 8, 9, 1123.

[2] Cheng Yew Leong. 2019. Fault detection and diagnosis of air handling unit: a review. In MATEC Web of Conferences. Volume 255. EDP Sciences, 06001.

[3] Leslie K Norford et al. 2002. Demonstration of fault detection and diagnosis methods for air-handling units. HVAC\&R Research, 8, 1, 41-71.

[4] Jessica Granderson et al. 2020. Building fault detection data to aid diagnostic algorithm creation and performance testing. Scientific data, 7, 1, 1-14.

[5] Chuanxing Geng, Sheng-jun Huang, and Songcan Chen. 2020. Recent advances in open set recognition: a survey. IEEE transactions on pattern analysis and machine intelligence.

[6] Jingyun Jia and Philip K Chan. 2021. Self-supervised detransformation autoencoder for representation learning in open set recognition. arXiv preprint arXiv:2105.13557.

[7] Walter J Scheirer, Anderson de Rezende Rocha, Archana Sapkota, and Terrance E Boult. 2012. Toward open set recognition. IEEE transactions on pattern analysis and machine intelligence, $35,7,1757-1772$.

[8] Lalit P Jain, Walter J Scheirer, and Terrance E Boult. 2014. Multi-class open set recognition using probability of inclusion. In European Conference on Computer Vision. Springer, 393-409.

[9] Hakan Cevikalp. 2016. Best fitting hyperplanes for classification. IEEE transactions on pattern analysis and machine intelligence, 39, 6, 1076-1088.

[10] Marcel Dix. 2021. A three-step machine learning pipeline for detecting and explaining anomalies in the time series of industrial process plants. In The International Conference on Deep Learning, Big Data and Blockchain. Springer, $15-26$.

[11] Anh Nguyen, Jason Yosinski, and Jeff Clune. 2015. Deep neural networks are easily fooled: high confidence predictions for unrecognizable images. In Proceedings of the IEEE conference on computer vision and pattern recognition, 427-436.

[12] Abhijit Bendale and Terrance E Boult. 2016. Towards open set deep networks. In Proceedings of the IEEE conference on computer vision and pattern recognition, 1563-1572.

[13] Poojan Oza and Vishal M Patel. 2019. C2ae: class conditioned auto-encoder for open-set recognition. In Proceedings of the IEEE/CVF Conference on Computer Vision and Pattern Recognition, 2307-2316.

[14] M Tonmoy et al. 2021. Hierarchical self attention based autoencoder for openset human activity recognition. arXiv preprint arXiv:2103.04279.

[15] Ryota Yoshihashi et al. 2019. Classification-reconstruction learning for openset recognition. In Proceedings of the IEEE/CVF Conference on Computer Vision and Pattern Recognition, 4016-4025.

[16] VDI/VDE. 2015. Formalised process descriptions - concept and graphic representation. In VDI/VDE Publication 3682, Blatt 1:2015-05.

[17] Fei-Niu Yuan et al. 2019. Theories and applications of auto-encoder neural networks: a literature survey. Chinese fournal of Computers, 42, 203-230.

[18] Marcel Dix et al. 2021. Anomaly detection in the time-series data of industrial plants using neural network architectures. In Proceedings of The 7th IEEE International Conference on Big Data Computing Service and Machine Learning Applications.

[19] Guoqiang Peter Zhang. 2000. Neural networks for classification: a survey. IEEE Transactions on Systems, Man, and Cybernetics, Part C (Applications and Reviews), 30, 4, 451-462.

[20] Haitao Wang. 2016. Application of residual-based ewma control charts for detecting faults in variable-air-volume air handling unit system. Fournal of Control Science and Engineering, 2016. 